\title{
Graphene Nanomaterials: A Comprehensive Review
}

Priya Bhardwaj ${ }^{1 *}$, Upendra Kumar Sharma ${ }^{1}$, Saurabh Savita ${ }^{1}$ and Abha Mishra ${ }^{2}$

${ }^{1}$ Department of Pharmaceutics, Institute of Pharmacy, Bundelkhand University Jhansi - 284128, Uttar Pradesh, India

${ }^{2}$ Department of Medicinal Chemistry, NIPER, Bijnor Road Lucknow - 226002, Uttar Pradesh, India

DOI: $10.36347 /$ sajp.2020.v09i09.005

| Received: 06.09.2020 | Accepted: 14.09.2020 | Published: 17.09.2020

*Corresponding author: Ms. Priya Bhardwaj

Abstract

Review Article

Graphene is a material which is two dimensional, sp2 hybridized, organized in a hexagonal lattice. It is a miracle matter in the planet. Graphene derivative in developing discipline has seen tremendous evolution. The multidisciplinary attributes of graphene such as considerable surface area, thermal steadiness, and electrical conductivity attract immense contemplation at the moment. Nanomaterials based on Graphene are growing as a result of characterization, synthesis and large-scale manufacture of graphene. Graphene has portrayed a deep-rooted representation in the drug-delivery system. It's an assertive matter whenever it makes content mixed with other. In the present article, we review the utilizes of graphene in different sorts of drug delivery in addition to biological application.

Keyword: Graphene, synthesis, biocompatibility, drug delivery.

Copyright @ 2020: This is an open-access article distributed under the terms of the Creative Commons Attribution license which permits unrestricted use, distribution, and reproduction in any medium for non-commercial use (NonCommercial, or CC-BY-NC) provided the original author and source are credited.

\section{INTRODUCTION}

Graphene is leanest and potent material in the nature [1], Graphene derivative possess sets of achievements in the field of biomedical science as well as technology owing to its exceptional properties such as abundant surface area, electrical conductivity, exceptional mechanical strength, biocompatibility in addition to permeability enhancing virtue $[2,3]$.

In biomedical science, graphene has newly been spotted due to its competence to scavenge free radicals. Nanomaterial of graphene had appealed to the domain of tissue engineering as well as regenerative medicine on account of their surpassing therapeutic implementations. Graphene nanotubes and fullerences are the primary initial material of graphene. Carbon nanotubes construct a cylindrical hollow tube that can be enfolded by individual as well as multiple layers of carbon. Fullerences is a cylindrical structure that differs in the number of carbon atoms. Currently, permanent structures of fullerenes have been discovered that contain $60,70,72,76,78$, or 84 atoms. Fullerences properties shift harmonizing to their organizational frame of the atom. Graphene acts as initial fabric for carbon nanotubes and fullerences. The role of graphene in addition to its derivative is multifaceted; they may characterize as a nanocarrier or reinforcing matters along with are worthwhile designed for drug delivery system. The graphene-based formulation has been scrutinized in cell lines medically for toxic window in preclinical models depending on duration and dosage form. The alteration in the chemical constitution of native graphene material through blending in addition to oxidizing the surface has intensified the organic action of graphene formation. The planar framework of graphene accompanied by intense chemical reactiveness is brought into effective action to load miscellaneous pharmaceutical drugs as well as biomolecules in favor of maximum cleaning of free radicals. Hanns-Peter Boehm, a German chemist monitored graphene by means of microscopic analysis [4]. Graphene is a plentiful substance of carbon allotropes. Miscellaneous forms of carbon allotropes can be compounded by adapting the blend of sp, sp2 and sp3 hybridization. Carbon atoms having sp2 hybridization are ordered in a hexagonal lattice, there are various distinct morphologies, structure also properties. There are various groups of carbon material, arranged as $0 \mathrm{D}, 1 \mathrm{D}$, $2 \mathrm{D}$ and 3D. OD macrostructure are known as carbon dots, fullerences and graphene quantum dots. Onedimensional (1D) nanostructured materials, including nanotubes, nanofibers, and nanowires, 2D nanostructure are graphene, hexagonal boron nitride $(\mathrm{hBN})$, and metal dichalcogenides (MX2) [5-8].

Synthesis of graphene- in the early 1970s, graphene was synthesized through chemical deposition, Scotch tape and mechanical exfoliation technique to 
Priya Bhardwaj et al., Sch Acad J Pharm, September, 2020; 9(9): 278-282

yield high-quality graphene. The fabrication of graphene on a substantial degree can be accomplished exercising chemical synthesis, mechanical synthesis and chemical exfoliation process [9]. Additional paths for the mass-production of graphene incorporates chemical vapour deposition, pyrolysis and epitaxial growth.

\section{Characteristics and feasibility of graphene}

[1] Physicochemical and mechanical characteristics: Graphene is a plane film like construction and repeat in a regular hexagonal framework [10]. 2D material of graphene is one atom wide. The tensile firmness of graphene is $130 \mathrm{GPa}$ with surface area of $2636 \mathrm{~m}^{2} / \mathrm{g}$ as compared to nanotube of single walled carbon, which is around $1000 \mathrm{~m}^{2} / \mathrm{g}$ [11]. The graphene holds bond length of carbon- carbon is nearby $0.142 \mathrm{~nm}$ in addition to interplanar spacing of $0.335 \mathrm{~nm}[12]$. Graphene is the toughest matter with the tensile strength of $130 \mathrm{GPa}$ plus stiffness of $1 \mathrm{TPa}$ [13]. It designates that one square meter of graphene layer may well uphold four $\mathrm{kg}$ of material [14]. There are two arrangements of graphite, which include alpha and beta. Alpha hexagonal is close to $\mathrm{ABAB}$ orchestration on graphene sheet at the same time as beta hexagonal is rhombohedral $\mathrm{ABCABC}$ organization. The stretch of $\mathrm{c}$ -c $\sigma$ bond is ca.1.42 $\AA$ apart. The crystal framework can identify both structures. However, they hold comparable physical properties. In structure of alpha graphite, the interplanar spacing is $0.34 \mathrm{~nm}$ and the parallel distance is $0.67 \mathrm{~nm}$ [15]. There is a weak Van Der Waals force of attraction within the sheets of graphite, it is the weakest attraction and causes graphite to be soft, such as the lead of a pencil, and it can be fractured effortlessly. In favor of the chemical reaction, graphene comprises of carbon, holds two faces meant for the chemical reaction by its two-dimensional construction. The edge or side of graphene is chemically extremely reactive, and it got burned at temperature of $350{ }^{\circ} \mathrm{C}$ [16]. Graphene holds the properties of high opacity, high chemical reactiveness in addition to unparalleled thermal conductivity [17], functionalize readily and posses outstanding biocompatibility. Taking into consideration their matchless properties, it makes appealing to the academicians as well as scientists to reveal the implementations of graphene. One of the applicable domains for graphene is the biomedical application, and it comprises biosensing, bio-targeting, bioimaging in addition to further medical applications. The majority of the graphene-based matters are favored owing to its prominent functional groups such as graphene oxide and $\mathrm{N}$-graphene, are exceptionally applicable moreover efficacious.

[2] Antioxidative properties of graphene: Graphene impacts big effort on biomedical sciences. The smallest difference in the magnitude of graphene can root oxidative stress and or deliver an anti-oxidant effect in the biological system. The antioxidant attribute of graphene stalks from the sp2-hybridized carbon network and entails the creation of free radical adducts, thereby neutralizing free radicals [18]. Reactive oxygen species (ROS)-mediated oxidative stress in cells due to graphene appears on account of surface area normalization by proteins or surfactants [19], or contamination by hetero atoms or transition-metal ions that materializes in the course of the synthesis [20].

Biomedical application of graphene: The application of graphene as well as its derivative like graphene flakes, graphene oxide and reduced graphene oxide can be flourishingly soiled in biomedical sciences.

[1] Antibacterial properties of graphene: Although outstanding biocompatibility is noteworthy for considering a biomaterial. Moreover, its consequent scaffold successfully, the scaffold's competency not to spoil the standard habitat of the oral cavity is additionally necessary, which is primarily accomplished via inhibition of surface-level bacterial spread. This material essentially be able to assist cell adhesion as well as proliferation [21]. With other traits of graphene, the antibacterial properties be based on concentration, physical plus chemical properties of graphene and factor of bacteria soiled in investigation [22, 23]. Graphene nanocomposite holds the maximum potential in opposition to gram positive as well as gram-negative bacteria. However, usually against bacteria effects entering cell membrane and tamper with the bacterial function. Graphene sheet that is immersed in suspension can separate the bacteria from nutrient-rich surrounding environment [24] while function of graphene oxide can be effortlessly processed and competently execute water dispersion. However, these are advantageous properties of graphene oxide. Graphene oxide nanosheets antibacterial pursuit relies heavily on their shape. Substantial surface area of graphene sheet produces a highly antibacterial effect against E. Coli, and also reduces the pathogenetic capacity in the teeth depending on the presence of graphene oxide [25, 26]. Graphene as well as some of its joint structure also work against bacterial biofilm, other than single bacteria [27]. Relating to the antibacterial properties previously illustrated opposite Gram-positive and Gram-negative bacteria, this is also dose-dependent as well. While high graphene oxide concentrations restrain the establishment of Gram-positive and Gram-negative bacteria biofilms, low graphene oxide concentrations in reality intensify their formation, coining an absolutely adverse reaction to the planned [28]. While it may seem like it has an anti-bacterial effect on bacteria itself, it also has on biofilm bacteria itself. It has been investigated that less than $50 \mu \mathrm{g} / \mathrm{mL}$ graphene oxide in a nutrient medium solution has no antimicrobial activity and in fact, intensifies bacterial proliferation by acting as a biofilm itself. However, GOpolyoxyalkyleneamine, which is two antibacterial molecules in the equivalent concentration, displays antibacterial pursuit where bacteria are cultivated in 
phosphate-buffered saline solution [29, 30]. Graphene in dentistry, its antibacterial advantages are associated to the environment of the tests, especially the class of material and size of particles, concentration and state, as well as the strain of bacteria and medium used. In addition to all of this, the results on the antibacterial effects of graphene in the oral cavity are additionally dependent on the host susceptibility, diets and habits that could lead to a break in the balance of the oral microenvironment [31]. Although the antibacterial activity of graphene appears to be favorable, it is all relative and cannot yet be generalized.

[2] Antimicrobial activity: Graphene has been used for antimicrobial pursuit, display the efficacious antimicrobial effect. Sharp edges of graphene oxide can break up the cell membrane of gram positive and gramnegative bacteria. If it is more resistant to graphene oxide then less damage will be caused by the sharp edges. The rGO nanowalls will produce more toxicity to the bacteria than the nanowalls of GO. To sharpen the edges of GO and shift of charge in the course of the interaction with bacteria, GO can be adapted in order to boost the antimicrobial activity. Some investigations to establish the antimicrobial characteristic were organized by Liu et al. [32] and it was prophesied that the antimicrobial action of graphene-based material is hangs on different aspects, and the leading factors are generating from the membrane and oxidative stress. From the investigations handled by Kang et al. [33], it was established that the highly purified narrow diameter graphene can be used to damage the cell with direct cell contact to release the intracellular content.

[3] Anti-tumour drug therapies: Choroidal melanoma may diminished survival rate of five years due to high chance of tumor metastasis. The usual medication does not boost survival ratio and may additionally have side effects. In antitumor drug treatments, graphene may cater an prospect for controlled and targeted release. Modified graphene oxide accompanied by biocompatible polymer is immensely used in drug delivery [34, 35]. However, the implementation of graphene-based material is restricted by other flaws such as hazard residual synthesis, complicated synthesis protocol come from the chemical oxidation and reduction process. To improve the drug therapy, graphene used accompanied by polymer that is better capable to target tumor [36].

[4] Stem cells and tissue engineering: Graphene derivative can be utilized for tissue engineering because of its multiple properties used by the experts in different shorts of medical application. In this situation a number of prominent considerations are essential to be thought over which embrace cellular, morphological as well as physiological organization. Stem cells is principal region for tissue engineering which holds the prospective of self-renewal. For the applicable utilization of stem cells, some of the precise control needs to be thought over which are biochemical and physical environmental. Graphene can be the finest choice to promote the stem cells attachment, growth, viability and differentiation. It can create various forms of stem cell like embryonic stem cells, induced pluripotent stem cells, mesenchymal stem cells and neural stem cells due to its conspicuous biocompatibility in addition to its tunable functionalities [37]. Hu et al. [38] have professed that GO can be universal adhesive for the attachment of protein. Employing bovine serum albumin-coated GO, they are used to assemble metallic nanoparticles such as gold, platinum, palladium and silver. Graphene has been used with numerous interactions by specific chemical groups to adsorb the protein. Besides, rGo-chitosan substrates can be used for human mesenchymal stem cell differentiation with minimal cytotoxicity of $0.1 \mathrm{mg} / \mathrm{mL}$. Solanki et al. [39] formulated the arrays of graphenenanoparticle hybrid structure to notify the differentiation and to target the human neural stem cells by the assembly of positively charged silicon nanoparticle on the glass substrates with GO deposit and coated with an extracellular matrix protein laminin to boost adhesion and growth of human neural stem cells [40]. The domain step with the stem cells is to monitor differentiation with the stage processes that can be done by immune-techniques. Surface-enhanced Raman Spectroscopy has been used to distinguish the undifferentiated and differentiated stem cells using graphene-nanoparticle, while the outcome of undifferentiated stem cell shows a high percentage of $\mathrm{C}=\mathrm{C}$ bonds compared to differentiated stem cell [41].

\section{CONCLUSION}

Graphene has been used in diverse models of drug delivery on account of its specific surface and mechanical properties of material. Successfully developed therapies with graphene such as drug delivery, tissue engineering, gene therapy and bioimaging are now well-established and published. In the field of research, many achievements on graphenebased systems are prosperously instituted. However, there are a number of barriers need to be attended. In the future Graphene-based nanomaterial have remarkable scope for various research fields of biomedical and therapeutics.

\section{REFERENCE}

1. Geim AK, Novoselov KS. The rise of graphene, Nanoscience and Technology: a Collection of Reviews from Nature Journals, World Scientific. 2010; 11-19.

2. Wu ZS, Ren W, Gao L, Liu B, Jiang C, Cheng HM: Synthesis of high-quality graphene with a pre-determined number of layers. 2009; 47(2): 493-99.

3. Zhao J, Pei S, Ren W, Gao L, Cheng HM: Efficient preparation of large-area graphene oxide sheets for transparent conductive films. 2010; 4(9): 5245-52. 
4. Boehm HP: Graphene-how a laboratory curiosity suddenly became extremely interesting. Angew. Chem., Int. Ed. 2010; 49(49): 9332-35.

5. Huang X, Yin Z, Wu S, Qi X, He Q, Zhang Q, Yan Q, Boey F, Zhang H: Graphene-based materials: synthesis, characterization, properties, and applications. 2011; 7(14): 1876-1902.

6. Neto AC, Guinea F, and Peres NM. Drawing conclusions from graphene. J Physics world. 2006; 19(11): 33.

7. Eda G, Chhowalla M. chemically derived graphene oxide: towards large area thin-film electronics and optoelectronics. J Adv Mater. 2010; 22(22): 23922415.

8. Rao CNR, Sood AK, Subrahmanyam KS, Govindaraj A: Graphene: the new two-dimensional nanomaterial. 2009; 48(42): 7752-77.

9. Yi M, Shen Z: A review on mechanical exfoliation for the scalable production of graphene. J Mater Chem A. 2015; 3(22): 11700-15.

10. Cooper DR, Anjou BD, Ghattamaneni N, Harack B, Hilke M, Horth A, Majis N, Massicotte M, Vandsburger L, Whiteway E, Yu V: Experimental review of graphene, ISRN condens, Matter Phys. 2012; 1-56.

11. Biswas C, Lee YH: Graphene versus carbon nanotubes in electronic devices, Adv Funct Mater. 2011; 21: 3806-26.

12. Heyrovska R. Atomic structures of graphene, Benzene and methane with bond lengths as sums of the single, double and resonance bond radii of carbon, eprint arXiv: 0804.4086, General Physics [physics.gen-ph] 2008; 1-4.

13. Lee C, Wei X, Kysar JW, Hone J. Measurement of the elastic properties and intrinsic strength of monolayer graphene. Science. 2008; 80(321): 3858.

14. Royal THE, Academy SOF. Sciences, Graphene scientific background on the nobel prize in physics, R. Swedish Acad. Sci. 2010; 50005: 0-10.

15. Wong SC, YEOU9CgxzmgC.pdf, 2007; 1-7.

16. Eftekhari A, Jafarkhani P. Curly Graphene with Specious Interlayers Displaying Superior Capacity for Hydrogen Storage; 2013.

17. Balandin AA, Ghosh S, Bao W, Calizo I, Teweldebrhan D, Miao F, Lau CN. Superior thermal conductivity of single-layer graphene, Nano Lett. 2008; 8: 902-7.

18. Qiu Y, Wang Z, Owens AC, Kulaots I, Chen Y, Kane AB, Hurt RH: Antioxidant chemistry of graphene-based materials and its role in oxidation protection technology. Nanoscale. 2014; 6(20): 11744-55.

19. Chen B, Liu M, Zhang L, Huang J, Yao J, Zhang Z. Polyethylenimine functionalized graphene oxide as an efficient gene delivery vector. J Mater Chem. 2011; 21(21): 7736-41.

20. Chng EL, Sofer Z, Pumera M. Cytotoxicity profile of highly hydrogenated graphene. Chem. - Eur. J. 2014; 20(21): 6366-73.
21. Pinto AM, Goncalves IC, Magalhaes FD. Graphene-based materials biocompatibility: a review 2013; 111: 188-202.

22. Hu W, Peng C, Luo W, Lv M, Li X, Li D, Huang Q, Fan C. Graphene-based antibacterial paper. 2010; 4 (7): 4317-23.

23. Liu S, Zeng TH, Hofmann M, Burcombe E, Wei J, Jiang R, Kong J, Chen Y. Antibacterial activity of graphite, graphite oxide, graphene oxide, and reduced graphene oxide: membrane and oxidative stress. 2011; 5(9): 6971-80.

24. Akhavan O, Ghaderi E, Esfandiar A. Wrapping bacteria by graphene nanosheets for isolation from environment, reactivation by sonication, and inactivation by near-infrared irradiation, J Phys Chem B. 2011; 115(19): 6279-88.

25. Liu S, Hu M, Zeng TH, Wu R, Jiang R, Wei J, Wang L, Kong J, Chen Y. Lateral dimensiondependent antibacterial activity of graphene oxide sheets. 2012; 28 (33): 12364-72.

26. He J, Zhu X, Qi Z, Wang C, Mao X, Zhu C, He Z, Li M, Tang Z. Killing dental pathogens using antibacterial graphene oxide. 2015; 7(9): 5605-11.

27. Karatan E, Watnick P. Signals, regulatory networks, and materials that build and break bacterial biofilms. J Microbial Mol Biol Rev. 2009; 73 (2): 310-47.

28. Song C, Yang CM, Sun XF, Xia PF, Qin J, Guo $\mathrm{BB}$, Wang SG. Influences of graphene oxide on biofilm formation of gram-negative and grampositive bacteria. 2018; 25(3): 2853-60.

29. Guazzo R, Gardin C, Bellin G, Sbricoli L, Ferroni L, Ludovichetti FS, Piatteli A, Antoniac L, Bressan E, Zavan B: Graphene-based nanomaterials for tissue engineering in the dental field. 2018; 8(5): 349.

30. Wu PC, Chen HH, Chen SY, Wang WL, Yang KL, Huang CH, Kao HF, Chang JC, Hsu CL, Wang JY, Chou TM, Kuo WS. Graphene oxide conjugated with polymers: a study of culture condition to determine whether a bacterial growth stimulant or an antimicrobial agent? 2018; 16 (1): 1.

31. Wade WG. The oral microbiome in health and disease. J Pharm Res. 2013; 69(1): 137-43.

32. Liu S, Zeng TH, Hofmann M, Burcombe E, Wei J, Jiang R. Antibacterial Activity of Graphite, Graphite Oxide, Graphene Oxide and Reduced Graphene Oxide: Membrane and Oxidative Stress. 2011; 6971-80.

33. Kang S, Pinault M, Pfefferle LD, Elimelech M. Single-walled carbon nanotubes exhibit strong antimicrobial activity, Langmuir. 2007; 23: 867073.

34. Huang $P$, Wang S, Wang X, Shen G, Lin J, Wang Z, Guo S, Cui D, Yang M, Chen X. Surface functionalization of chemically reduced graphene oxide for targeted photodynamic therapy. J Biomed Nanotechnol. 2015; 11: 117. 
35. Zhi X, Fang H, Bao C, Shen G, Zhang J, Wang K, Guo S, Wan T, Cui D: Biomaterials. 2013; 34: 5254.

36. Danhier F, Feron O, Préat V. To exploits the tumor microenvironment: Passive and active tumor targeting of nanocarriers for anti-cancer drug delivery. J Controlled Release. 2010; 148: 135.

37. Chen GY, Pang DP, Hwang SM, Tuan HY, Hu YC: A graphene-based platform for induced pluripotent stem cells culture and differentiation, Biomaterials. 2012; 33: 418-27.

38. Hu W, Peng C, Lv M, Li X, Zhang Y, Chen N, Fan C, Huang Q: Protein corona-mediated mitigation of cytotoxicity of graphene oxide, ACS Nano. 2011; 5: 3693-3700.

39. Solanki A, Shah S, Yin PT, Lee KB. Nanotopography-mediated reverse uptake for siRNA delivery into neural stem cells to enhance neuronal differentiation. Sci Rep. 2013; 3: 1-7.

40. Yin PT, Shah S, Chhowalla M, Lee KB. Design, synthesis, and characterization of graphenenanoparticle hybrid materials for bioapplications. Chem Rev. 2015; 115: 2483-2531.

41. Ramm Sander P, Hau P, Koch S, Schütze K, Bogdahn U, Kalbitzer HR, Aigner L: Stem cell metabolic and spectroscopic profiling. Trends Biotechnol. 2013; 31: 204-13. 